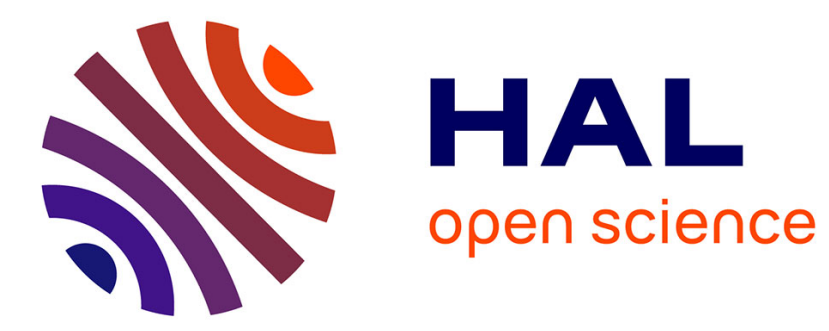

\title{
Object digitization up to a translation
}

\author{
Loïc Mazo, Étienne Baudrier
}

\section{To cite this version:}

Loïc Mazo, Étienne Baudrier. Object digitization up to a translation. Journal of Computer and System Sciences, 2018, 95, pp.193-203. 10.1016/j.jcss.2017.08.001 • hal-01384377v2

\section{HAL Id: hal-01384377 \\ https://hal.science/hal-01384377v2}

Submitted on 28 Aug 2017

HAL is a multi-disciplinary open access archive for the deposit and dissemination of scientific research documents, whether they are published or not. The documents may come from teaching and research institutions in France or abroad, or from public or private research centers.
L'archive ouverte pluridisciplinaire HAL, est destinée au dépôt et à la diffusion de documents scientifiques de niveau recherche, publiés ou non, émanant des établissements d'enseignement et de recherche français ou étrangers, des laboratoires publics ou privés. 


\title{
Object digitization up to a translation
}

\author{
Loïc Mazo, Étienne Baudrier \\ ICube-UMR 7357, 300 Bd Sébastien Brant - CS 10413 \\ 67412 Illkirch Cedex France
}

\begin{abstract}
This paper presents a study on the set of the digitizations generated by all the translations of a planar body on a square grid. First the translation vector set is reduced to a bounded subset, then the dual introduced in [BM16] linking the translation vector to the corresponding digitization is proved to be piecewise constant. Finally, a new algorithm is proposed to compute the digitization set using the dual.
\end{abstract}

Keywords: digitization, plane curve, translation, dual representation

\section{Introduction}

For a given planar body digitization method, the result depends on the object relative position with respect to the digitization grid. As a result, distinct digital sets can be obtained. This variability may influence the reconstruction of the geometrical and topological attributes of the planar body. For instance, conditions have been given to preserve the topology during the digitization step [TR02, SK05].

This paper focuses on the effect of the grid translations on the digitized object. This issue has been studied on some geometrical primitives, i.e. the straight segments and the discs. Straight segment digitizations have been discussed in function of the straight segment slope and its vertical position. The function giving the digital straight segment from these two inputs is known as the preimage. Several properties have been proved on the preimage and it is widely used, e.g. for digital straight segment recognition [DS84]. The number of oval and disc digitizations in function of their radius up to a translation was studied in [Ken48, Nag05, HŽ06]. The number of digital discs including exactly $\mathrm{N}$ points was treated in [HŽ07] and an asymptotic bound on this number was given in [HŽ16]. Our study follows a previous work [BM16] which focused on function graphs digitizations.

After introducing the dual definition in Section 2, its structure is investigated in Section 3 and it is proved to be piecewise constant. Two algorithms

Email address: mazo@unistra.fr (Loïc Mazo, Étienne Baudrier) 
taking the dual as input are presented in Section 4. The first one computes the digitization of a planar body for a particular position of the grid while the second one propagates the output of the former to obtain the whole family of the digitizations.

\section{Definition of the digitization dual}

Let us consider a connected set $S$ in $\mathbb{R}^{2}$ whose boundary is a simple closed (Jordan) curve $\Gamma$. Thanks to the Jordan curve theorem, we may assume a continuous map $f: \mathbb{R}^{2} \rightarrow \mathbb{R}$ such that $\Gamma$, resp. $S$, is implicitly defined by $\Gamma=\left\{x \in \mathbb{R}^{2} \mid f(x)=0\right\}$, resp. $S=\left\{x \in \mathbb{R}^{2} \mid f(x) \leq 0\right\}$.

The common methods to model the digitization of the set $S$ are closely related to each other. In this paper, we assume a Gauss digitization. This method associates to the set $S$ the grid points that lie inside $S$ or, equivalently, the binary image defined on $\mathbb{Z}^{2}$ whose 1 's are the points inside $S$. The aim of this paper is to represent and describe the set of the Gauss digitizations of $\Gamma$ obtained using the grids generated by the action of the translation group on the standard grid. Equivalently, we can consider a unique grid, the standard one, and let the group of translations acts on $S$. These two points of view are used in the article. Figure 1 exhibits these Gauss digitizations for the set $\mathcal{S}_{\text {astro }}$ bounded by the "stretched" astroid

$$
\left(\frac{x}{2}\right)^{2 / 3}+y^{2 / 3}=1
$$

We will use this shape to illustrate the notions and properties discussed throughout the article.

The Gauss digitization is a finite subset of $\mathbb{Z}^{2}$ and we are only interested in the relative positions of its elements. In other words, $\mathbb{Z}^{2}$ is viewed as a geometrical subset of the Euclidean plane without any preferential origin. Thus, rather than defining a mapping from the translation vectors to the digitizations of the translated body, we define a mapping $\tilde{\varphi}_{S}$ from the torus $\mathbb{R}^{2} / \mathbb{Z}^{2}$ to the equivalence classes under integral translations of the digitizations (the tilde sign is used throughout the article when the function domain is the torus). This mapping $\tilde{\varphi}_{S}$ is called the dual ${ }^{1}$ of the digitizations.

For any vector $u \in \mathbb{R}^{2}$, we write $\langle u\rangle$ for the fractional part of $u$, that is for the vector of $\mathbb{R}^{2}$ whose coordinates are in $[0,1)$ and such that $\langle\mathbf{u}\rangle \in u-\mathbb{Z}^{2}$. By extension, for any equivalence class $\mathbf{t} \in \mathbb{T}$, we write $\langle\mathbf{t}\rangle$ for the common vector $\langle u\rangle, u \in \mathbf{t}$. We use the vector $\langle\mathbf{t}\rangle$ as the canonical representative of the class t. We also set $S_{u}=-u+S: S_{u}$ is the translation with vector $-u$ of the set $S$ and is defined by $f(x+u) \leq 0$. This latter equation is also the new equation of the body $S$ when the grid is translated with the vector $u$. Then, the dual $\tilde{\varphi}_{S}$ is

\footnotetext{
${ }^{1}$ We call it "dual" for the translations act on the digitizations while the dual of the digitizations maps the translations to the digitizations.
} 


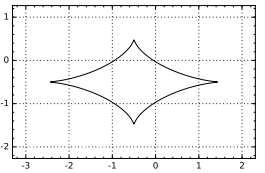

$u=(0.5,0.5)$

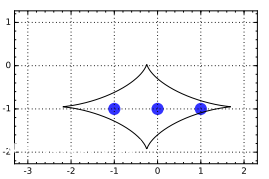

$u=(0.25,0.95)$

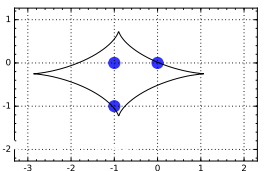

$u=(0.9,0.25)$

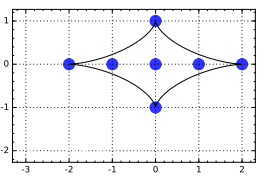

$u=(0,0)$

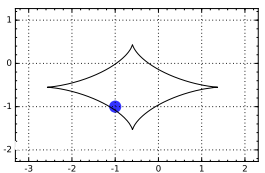

$u=(0.6,0.55)$

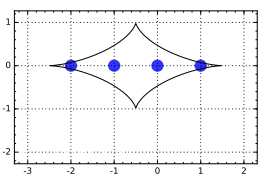

$u=(0.5,0)$

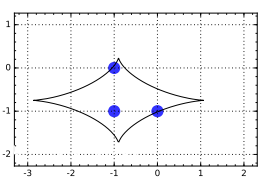

$u=(0.9,0.75)$

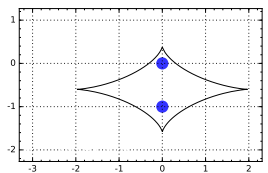

$u=(0,0.6)$

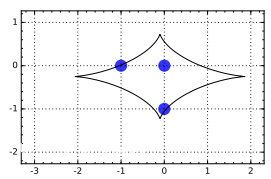

$u=(0.1,0.25)$

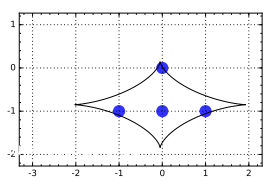

$u=(0.05,0.85)$

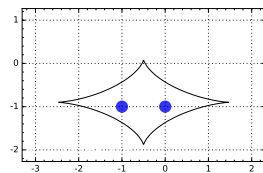

$u=(0.5,0.9)$

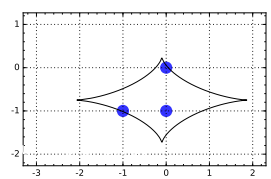

$u=(0.1,0.75)$

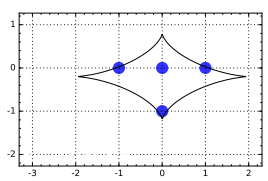

$u=(0,0.2)$

Figure 1: The thirteen Gauss digitizations of the sets defined by $\left(\left(x+u_{x}\right) / 2\right)^{2 / 3}+$ $\left(y+u_{y}\right)^{2 / 3} \leq 1,\left(u_{x}, u_{y}\right) \in \mathbb{R}^{2}$ (the first one is the empty set). The goal of the article is to give a representation of the mapping between the translation vector $\left(u_{x}, u_{y}\right)$ and the digitization.

such that, for any $\mathbf{t} \in \mathbb{T}$,

$$
\tilde{\varphi}_{S}(\mathbf{t})=\left\{S_{u} \cap \mathbb{Z}^{2} \mid u \in \mathbf{t}\right\}=\left[S_{\langle\mathbf{t}\rangle} \cap \mathbb{Z}^{2}\right],
$$

where $[\cdot]$ denotes the equivalent class under the integer translations.

The dual can be represented as a label image over the torus $\mathbb{T}$. An example of dual is shown in Fig. 2 (deployed torus) and Fig. 3. It is the dual of the set $\mathcal{S}_{\text {astro. }}$ Each point $u \in[0,1)^{2}$ in Fig. 2 corresponds to a translation and the color of this point corresponds to the digitization class $\left[S_{u} \cap \mathbb{Z}^{2}\right]$ : points having the same color in the dual correspond to translations giving the same digitization up to integer translations. 

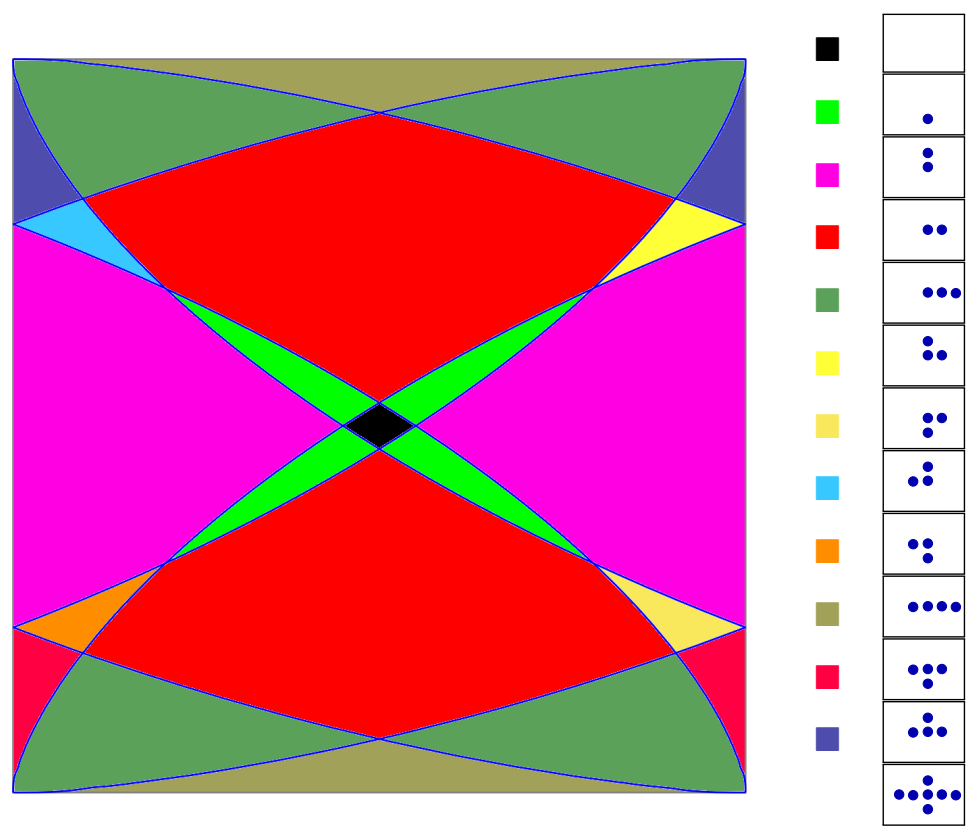

Figure 2: A deployed representation of the dual of $\mathcal{S}_{\text {astro. }}$ Note that the 7-points digitization region contains only one point, so it is not perceptible.

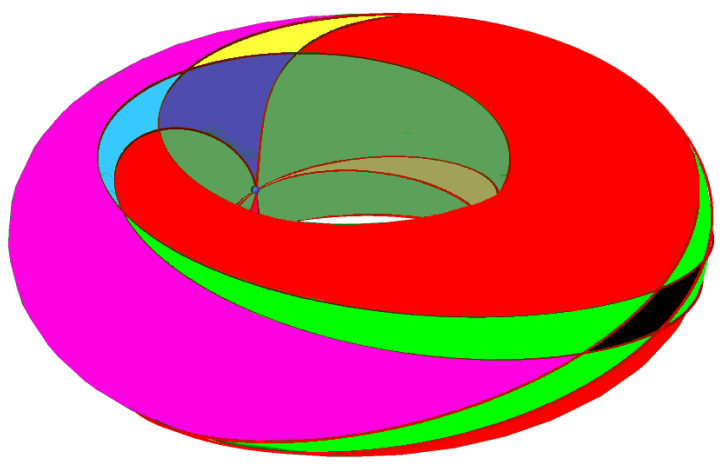

Figure 3: The dual of $\mathcal{S}_{\text {astro }}$. 
Remark 2.1. B. Nagy represents in [Nag05] regions of the translation vector set $[0,1)^{2}$ corresponding to distinct digitizations for the special case of the disc with radius 2. The dual of the disc with radius 2 is shown on Figure 4(a) (actually, rather a gradient image than the dual itself). One can see that Nagy's representation (Figure 4(b)) is a sketch (using straight lines) of the dual first octant.

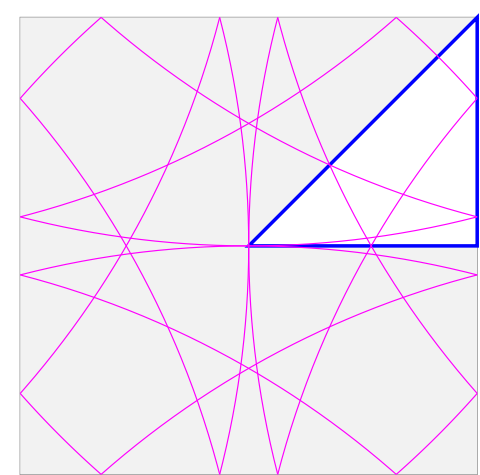

(a)

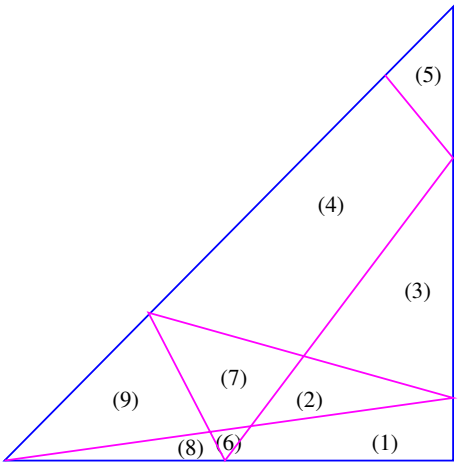

(b)

Figure 4: (a) Dual gradient image of a disc with radius 2. (b) Figure 4 in [Nag05].

\section{Properties}

The main property of the dual is that the plot of the curve $\Gamma$ on the torus $\mathbb{T}$ delineates regions on which the dual function $\tilde{\varphi}_{S}$ is constant. Before proving this property, we give some complementary definitions and notations related to the digitizations and the dual.

Firstly, we split the Gauss digitization in two parts with respect to the action of the translations. Thus, we define the grid boundary $\mathcal{B}$ as the set of grid points that lie in the (morphological) dilation of the boundary $\Gamma$ of $S$ by the unit square $(-1,0]^{2}$ :

$$
\mathcal{B}=\left(\Gamma \oplus(-1,0]^{2}\right) \cap \mathbb{Z}^{2},
$$

where $\oplus$ denotes the Minkowski sum (see Figure 5). It is plain that $\mathcal{B}$ contains all the points of $\mathbb{Z}^{2}$ whose value can change when we shift the set $S$ by a translation with a vector $u \in(-1,0]^{2}$ and that $\left(S \cap \mathbb{Z}^{2}\right) \backslash \mathcal{B}$, that we call the digitization core, contains the points that are in any digitization. In our set instance $S_{\text {astro, }}$, the core is empty, which results in an empty digitization (see Fig 1).

Secondly, we describe the plot of $\Gamma$ on $\mathbb{T}$ and its relation with the dual. Let $u \in \mathbb{R}^{2}$. We write $\lfloor u\rfloor$ for the vector whose coordinates are the floor of the 

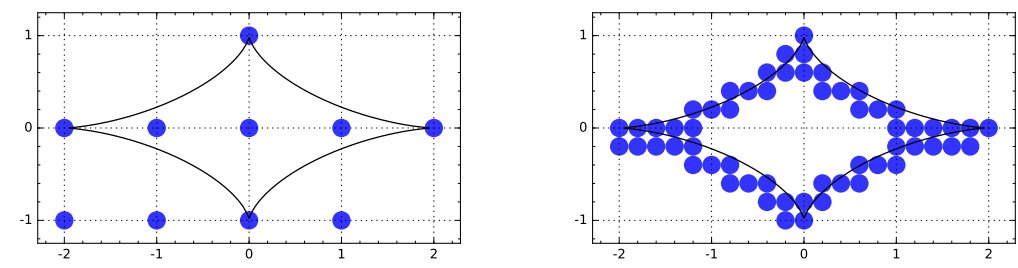

Figure 5: The grid boundary of $\mathcal{S}_{\text {astro }}$ at two resolutions $(\times 1$ and $\times 5)$.
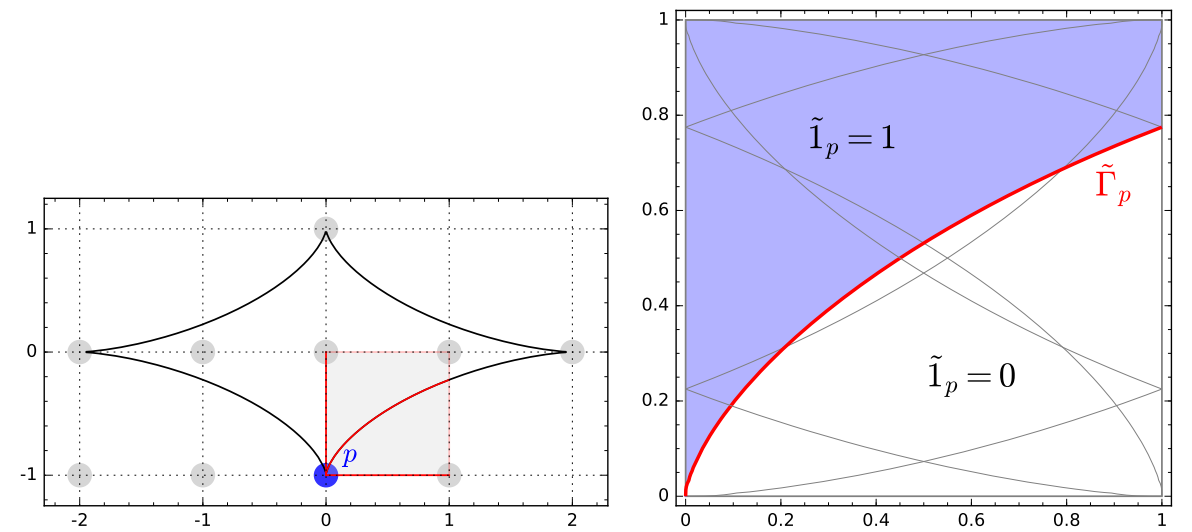

Figure 6: Left: a point $p$ in the grid boundary and the square $\{p\} \oplus[0,1)^{2}$. Right: the curve $\tilde{\Gamma}_{p}$ (in red) and the indicator function $\tilde{\mathbf{1}}_{p}$ (white: the region $\tilde{\mathbf{1}}_{p}=0$, blue: the region $\tilde{\mathbf{1}}_{p}=1$ ). The curve $\tilde{\Gamma}_{p}$ is included in the blue region.

coordinates of $u$. Thus $u=\langle u\rangle+\lfloor u\rfloor$. For describing the dual, it is useful to identify each restriction of the curve $\Gamma$ and the set $S$ to the unit squares with integer coordinates. Then, for any $p \in \mathbb{Z}^{2}$, we define the functions

$$
\begin{array}{rlrl}
f_{p}:[0,1) \rightarrow \mathbb{R} & \tilde{f}_{p}: \mathbb{T} & \rightarrow \mathbb{R} \\
u & \mapsto f(p+u), & \mathbf{t} & \mapsto f_{p}(\langle\mathbf{t}\rangle)=f(p+\langle\mathbf{t}\rangle) .
\end{array}
$$

When the origin of the coordinate system is set to $p$, the function $f_{p}$ makes it possible to describe the $\operatorname{arc} \Gamma_{p}$ of $\Gamma$ that is included in the grid square $p \oplus[0,1)^{2}$ by the equation $f_{p}=0$. Alike, $f_{p} \leq 0$ is the equation of $S \cap\left(p \oplus[0,1)^{2}\right)$ in this coordinate system. We denote by $\tilde{\mathbf{1}}_{p}$ the indicator function of the set $\tilde{f}_{p} \leq 0$ on the torus $\mathbb{T}$. We also define $\tilde{\Gamma}$ and $\tilde{\Gamma}_{p}$ as the projections of $\Gamma$ and $\Gamma_{p}$ on the $\mathbb{T}$. Hence, $\tilde{f}_{p}=0$ is an implicit equation of $\Gamma_{p}$. These definitions are illustrated by Figure 6.

Then, we show that the plot of $\Gamma$ on the torus is the superposition of the plots of the implicit functions $\tilde{f}_{p}=0$, that is, the superposition of the arcs of $\Gamma$ delimited by the squares $\{p\} \oplus[0,1)^{2}, p \in \mathcal{B}$. Alike, the digitizations can be computed by means of the indicator functions $\tilde{\mathbf{1}}_{p}$, that is, by the superposition 
of the parts of $S$ delimited by the squares $\{p\} \oplus[0,1)^{2}, p \in \mathcal{B}$.

Proposition 3.1. The plot $\tilde{\Gamma}$ of $\Gamma$ on $\mathbb{T}$, defined by $\tilde{\Gamma}=\{\mathbf{t} \in \mathbb{T} \mid \mathbf{t} \cap \Gamma \neq \emptyset\}$, and the dual of the digitizations $\tilde{\varphi}_{S}$ satisfy the following properties:

$$
\begin{gathered}
\tilde{\Gamma}=\bigcup_{p \in \mathcal{B}} \tilde{\Gamma}_{p}=\bigcup_{p \in \mathbb{Z}^{2}} \tilde{\Gamma}_{p} \\
\tilde{\varphi}_{S}(\mathbf{t})=\left[\left\{p \in \mathbb{Z}^{2} \mid \tilde{f}_{p}(\mathbf{t}) \leq 0\right\}\right]=\left[\left\{p \in \mathbb{Z}^{2} \mid \tilde{\mathbf{1}}_{p}(\mathbf{t})=1\right\}\right] \\
\operatorname{card}\left(\tilde{\varphi}_{S}(\mathbf{t})\right)=\sum_{p \in \mathbb{Z}^{2}} \tilde{\mathbf{1}}_{p}(\mathbf{t})=\sum_{p \in \mathcal{B}} \tilde{\mathbf{1}}_{p}(\mathbf{t})+\operatorname{card}\left(\left(S \cap \mathbb{Z}^{2}\right) \backslash \mathcal{B}\right) .
\end{gathered}
$$

Proof. (2) Let $\mathbf{t} \in \tilde{\Gamma}$. By definition of $\tilde{\Gamma}$, there exists $u \in \mathbf{t}$ s.t. $u \in \Gamma$. Then, $\lfloor u\rfloor \in \mathcal{B}$. Moreover,

$$
\begin{aligned}
u \in \Gamma & \Longleftrightarrow f(u)=0 \\
& \Longleftrightarrow f_{\lfloor u\rfloor}(\langle u\rangle)=0 \\
& \Longleftrightarrow \tilde{f}_{\lfloor u\rfloor}(\mathbf{t})=0 \\
& \Longleftrightarrow \mathbf{t} \in \tilde{\Gamma}_{\lfloor u\rfloor} .
\end{aligned}
$$

Thereby, we have $\tilde{\Gamma}=\bigcup_{p \in \mathcal{B}} \tilde{\Gamma}_{p}$ and by definition of $\mathcal{B}$, the set $\tilde{\Gamma}_{p}$ is empty whenever $p \notin \mathcal{B}$.

(3) Let $\mathbf{t} \in \mathbb{T}$. According to (1),

$$
\tilde{\varphi}_{S}(\mathbf{t})=\left[S_{\langle\mathbf{t}\rangle} \cap \mathbb{Z}^{2}\right]=\left[\left\{p \in \mathbb{Z}^{2} \mid f(p+\langle\mathbf{t}\rangle) \leq 0\right] .\right.
$$

Hence,

$$
\tilde{\varphi}_{S}(\mathbf{t})=\left[\left\{p \in \mathbb{Z}^{2} \mid \tilde{f}_{p}(\mathbf{t}) \leq 0\right\}\right]=\left[\left\{p \in \mathbb{Z}^{2} \mid \tilde{\mathbf{1}}_{p}(\mathbf{t})=1\right\}\right] .
$$

(4) Let $\mathbf{t} \in \mathbb{T}$. From (3), we derive that card $\left(\tilde{\varphi}_{S}(-\mathbf{t})\right)=\sum_{p \in \mathbb{Z}^{2}} \tilde{\mathbf{1}}_{p}(\mathbf{t})$.

Moreover, it is plain that $p+\langle\mathbf{t}\rangle \in S$ implies $p \in \mathcal{B} \cup S$.

We illustrate Prop. 3.1 by computing the region boundaries of the dual of the set $S_{\text {astro }}$ (Figure 3) with the following SAGE program. The result is shown in Figure 7.

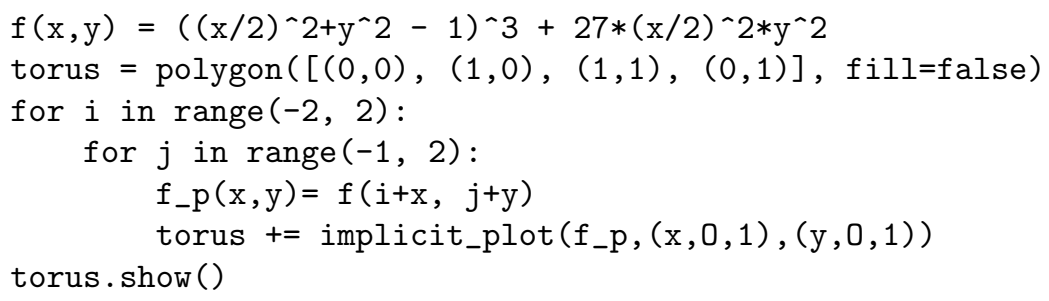




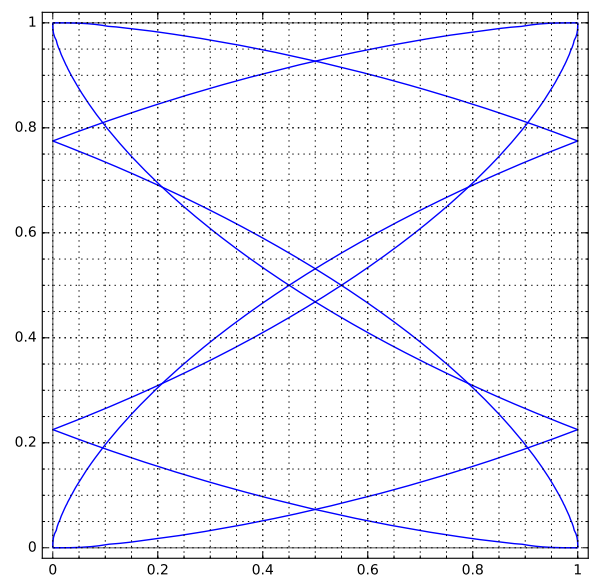

Figure 7: Region boundaries for the dual of the set instance $\mathcal{S}_{\text {astro }}$.

Eventually, we state and prove a technical lemma which is a key point in the proof of the subsequent theorem and propositions. In this lemma and in the sequel of the article, we denote by $B(z, r)$ the ball of center $z \in \mathbb{R}^{2}$ and radius $r>0$ relative to the infinite norm of $\mathbb{R}^{2}$.

Lemma 3.1. Let $\mathbf{t} \in \tilde{U}$ where $\tilde{U}$ is an open subset of $\mathbb{T} \backslash \tilde{\Gamma}$. Then, there exists $\varepsilon \in(0,1 / 2)$, such that $[B(\langle\mathbf{t}\rangle, \varepsilon)] \subseteq \tilde{U}$ and, for any vector $\mathbf{v} \in[B(\langle\mathbf{t}\rangle, \varepsilon)]$, the integer vector $q=\lfloor\langle\mathbf{v}\rangle-\langle\mathbf{t}\rangle+(1 / 2,1 / 2)\rfloor$ satisfies

$$
\forall p \in \mathbb{Z}^{2}, \operatorname{sign}\left(\tilde{f}_{p}(\mathbf{v})\right)=\operatorname{sign}\left(\tilde{f}_{p+q}(\mathbf{t})\right) .
$$

Note that $\|q\|_{\infty} \leq 1$ by definition.

Proof. We set $S^{+}=S \oplus B(0,1 / 2)$.

Let $\mathbf{t} \in \mathbb{T} \backslash \tilde{\Gamma}$. Then, for any $u \in \mathbf{t}, f(u) \neq 0$. Since $f$ is continuous, the sets $f>0$ and $f<0$ are open. Thereby, for any $u \in \mathbf{t} \cap S^{+}$, there exists $\varepsilon_{u} \in(0,1 / 2)$ such that the open ball $B\left(u, \varepsilon_{u}\right)$ does not intersect the curve $\Gamma$. We assume without loss of generality that $\left[B\left(u, \varepsilon_{u}\right)\right] \subseteq \tilde{U}$. As $S$ is bounded, we can set

$$
\varepsilon=\min _{u \in \mathbf{t} \cap S^{+}} \varepsilon_{u} .
$$

From the definition of $S^{+}$, we derive that no ball $B(u, \varepsilon), u \in \mathbf{t}$, intersects $\Gamma$. Hence, since $f$ is continuous, the sign of $f$ is constant on any ball $B(u, \varepsilon), u \in \mathbf{t}$.

We now consider a vector $\mathbf{v}$ in $[B(\langle\mathbf{t}\rangle, \varepsilon)]$. Then, for any $p \in \mathbb{Z}^{2}$, there exists $q \in \mathbb{Z}^{2}$ such that

$$
p+\langle\mathbf{v}\rangle \in B(p+q+\langle\mathbf{t}\rangle, \varepsilon) .
$$

In particular,

$$
\langle\mathbf{v}\rangle-\langle\mathbf{t}\rangle \in B\left(q, \frac{1}{2}\right) .
$$


In other words, $q=\lfloor\langle v\rangle-\langle t\rangle+(1 / 2,1 / 2)\rfloor$. Then, using the fact that the sign of $f$ is constant on any ball $B(u, \varepsilon), u \in \mathbf{t}$, we can state

$$
\begin{aligned}
\tilde{f}_{p}(\mathbf{v}) \leq 0 & \Longleftrightarrow f(p+\langle\mathbf{v}\rangle) \leq 0 \\
& \Longleftrightarrow f(p+q+\langle\mathbf{t}\rangle) \leq 0 \Longleftrightarrow \tilde{f}_{p+q}(\mathbf{t}) \leq 0 .
\end{aligned}
$$

We now establish the main property of the dual.

Theorem 1. Let $S$ be a compact subset of $\mathbb{R}^{2}$ whose boundary $\Gamma$ is a Jordan curve. The dual $\tilde{\varphi}_{S}$ is constant on the connected components of $\mathbb{T} \backslash \tilde{\Gamma}$.

Proof. Let $\mathbf{t} \in \mathbb{T} \backslash \tilde{\Gamma}$. From Lemma 3.1, there exists a neighborhood $\tilde{N}$ of $\mathbf{t}$ such that, for any $\mathbf{v} \in \tilde{N}, \forall p \in \mathbb{Z}^{2}, \operatorname{sign}\left(\tilde{f}_{p}(\mathbf{v})\right)=\operatorname{sign}\left(\tilde{f}_{p+q}(\mathbf{t})\right)$ where $q$ does not depend on $p$. Therefore, for any $\mathbf{v} \in \tilde{N}$, the sets $\left\{p \in \mathbb{Z}^{2} \mid \tilde{f}_{p}(\mathbf{v}) \leq 0\right\}$ and $\left\{p \in \mathbb{Z}^{2} \mid \tilde{f}_{p}(\mathbf{t}) \leq 0\right\}$ are congruent modulo integer translations. We derive that $\tilde{\varphi}_{S}$ is locally constant on $\mathbb{T} \backslash \tilde{\Gamma}$. Thereby, $\tilde{\varphi}_{S}$ is constant on any connected component of $\mathbb{T} \backslash \tilde{\Gamma}$.

Let us now study the converse situation of the one dealt with in Theorem 1: what happens when a boundary of the dual is crossed? To answer this question, we need to introduce two new curves on the torus $\mathbb{T}$, the outer equator $\mathrm{X}$ which is the quotient space of the $x$ axis of $\mathbb{R}^{2}$, and the prime meridian $\mathrm{Y}$ which is the quotient space of the $y$ axis of $\mathbb{R}^{2}$. Moreover, we also need to complete Theorem 1 by the following Lemma.

Lemma 3.2. For any $p \in \mathbb{Z}^{2}$, the function $\tilde{\mathbf{1}}_{p}$ is constant on the connected components of $\mathbb{T} \backslash\left(\tilde{\Gamma}_{p} \cup \mathrm{X} \cup \mathrm{Y}\right)$.

Proof. Let $p \in \mathbb{Z}^{2}$ and $\mathbf{t} \in \tilde{U}=\mathbb{T} \backslash\left(\tilde{\Gamma}_{p} \cup \mathrm{X} \cup \mathrm{Y}\right)$. We set $u=p+\langle\mathbf{t}\rangle$. From Lemma 3.1, there exists $\varepsilon \in(0,1 / 2)$ such that $[B(\langle\mathbf{t}\rangle, \varepsilon)] \subseteq \tilde{U}$ and, for any $\mathbf{v} \in[B(\langle\mathbf{t}\rangle, \varepsilon)], \operatorname{sign}\left(\tilde{f}_{p}(\mathbf{v})=\operatorname{sign}\left(\tilde{f}_{p+q_{\mathbf{v}}}(\mathbf{t})\right.\right.$ where $q_{\mathbf{v}}=\lfloor\langle\mathbf{v}\rangle-\langle\mathbf{t}\rangle+(1 / 2,1 / 2)\rfloor$. Since $[B(\langle\mathbf{t}\rangle, \varepsilon)]$ does not intersect $X$ nor $Y,\langle\mathbf{v}\rangle-\langle\mathbf{t}\rangle \in(-1 / 2,1 / 2)^{2}$. Thus, $q_{\mathbf{v}}=(0,0)$. We derive that $\tilde{\mathbf{1}}_{p}$ is constant on $[B(\langle\mathbf{t}\rangle, \varepsilon)]$. Thereafter, $\tilde{\mathbf{1}}_{p}$ is locally constant, which achieves the proof.

We now establish that crossing the curve $\tilde{\Gamma}$ on the torus $\mathbb{T}$ generally amounts to remove, or to add, a particular point in the digitization of the set. Moreover, crossing the prime meridian, resp. the outer equator, on the torus results in a unit horizontal, resp. vertical shift, on the parameter of the indicator functions (provided the crossing does not occur on a boundary point).

Proposition 3.2. Let $\mathbf{i}=(1,0), \mathbf{j}=(0,1)$ and $p \in \mathcal{B}$.

a) For any $\mathbf{t} \in \tilde{\Gamma}_{p} \backslash\left(\left(\bigcup_{q \neq p} \tilde{\Gamma}_{q}\right) \cup \mathrm{X} \cup \mathrm{Y}\right)$, there exists a neighborhood $\tilde{N}$ of $\mathbf{t}$ on which the functions $\tilde{\mathbf{1}}_{q}, q \neq p$, are constant and $\tilde{\mathbf{1}}_{p}$ is not constant. 
b) For any $\mathbf{t} \in \mathrm{Y} \backslash\left(\tilde{\Gamma}_{p} \cup X\right)$, there exists a neighborhood $\tilde{N}$ of $\mathbf{t}$ on which

$$
\left\{\begin{array}{l}
\tilde{\mathbf{1}}_{p}(\mathbf{v})=\tilde{\mathbf{1}}_{p}(\mathbf{t}) \text { if the abscissa of }\langle\mathbf{v}\rangle \text { is less than } 1 / 2, \\
\tilde{\mathbf{1}}_{p-\mathbf{i}}(\mathbf{v})=\tilde{\mathbf{1}}_{p}(\mathbf{t}) \text { otherwise }
\end{array}\right.
$$

c) For any $\mathbf{t} \in \mathrm{X} \backslash\left(\tilde{\Gamma}_{p} \cup Y\right)$, there exists a neighborhood $\tilde{N}$ of $\mathbf{t}$ on which

$$
\left\{\begin{array}{l}
\tilde{\mathbf{1}}_{p}(\mathbf{v})=\tilde{\mathbf{1}}_{p}(\mathbf{t}) \text { if the ordinate of }\langle\mathbf{v}\rangle \text { is less than } 1 / 2, \\
\tilde{\mathbf{1}}_{p-\mathbf{j}}(\mathbf{v})=\tilde{\mathbf{1}}_{p}(\mathbf{t}) \text { otherwise }
\end{array}\right.
$$

Proof. Let $p \in \mathcal{B}$.

a) We assume $\mathbf{t} \in \tilde{\Gamma}_{p}$ and $\mathbf{t} \notin\left(\left(\bigcup_{q \neq p} \tilde{\Gamma}_{q}\right) \cup \mathrm{X} \cup \mathrm{Y}\right)$. Since $\mathcal{B}$ is finite, we derive from Lemma 3.2 that there exists a neighborhood $\tilde{N}$ of $\mathbf{t}$ on which the functions $\tilde{\mathbf{1}}_{q}, q \neq p$, are constant and $\tilde{N} \cap X=\tilde{N} \cap Y=\emptyset$. Let $U$ be the neighborhood of $p+\langle\mathbf{t}\rangle$ whose projection on $\mathbb{T}$ is $\tilde{N}$. Since $\Gamma$ is a Jordan curve, the set $U$ intersects both the interior and the exterior of the set $S$. Then, $\tilde{\mathbf{1}}_{p}$ is not constant on $\tilde{N}$.

b) Figure 8 illustrates this part of the proof. Let $\mathbf{t} \in \mathrm{Y} \backslash\left(\tilde{\Gamma}_{p} \cup X\right)$. From Lemma 3.1, there exists $\varepsilon \in(0,1 / 2)$ such that $\tilde{N}=[B(\langle\mathbf{t}\rangle, \varepsilon)] \subseteq \mathbb{T} \backslash\left(\tilde{\Gamma}_{p} \cup X\right)$ and, for any $\mathbf{v} \in \tilde{N}, \tilde{\mathbf{1}}_{p}(\mathbf{v})=\tilde{\mathbf{1}}_{p+q}(\mathbf{t})$ with $q=\lfloor\langle\mathbf{v}\rangle-\langle\mathbf{t}\rangle+(1 / 2,1 / 2)\rfloor$. Let $\mathbf{v} \in \tilde{N}$. If the abscissa of $\langle\mathbf{v}\rangle$ is less than $1 / 2$, and taking into account that $\tilde{N}$ does not intersect $X$, we have $\langle\mathbf{v}\rangle-\langle\mathbf{t}\rangle \in[0,1 / 2) \times(-1 / 2,1 / 2)$. Hence, $q=0$. Otherwise, $\langle\mathbf{v}\rangle-\langle\mathbf{t}\rangle \in[1 / 2,1) \times(-1 / 2,1 / 2)$. Then $q=\mathbf{i}$.

c) The proof is similar to the proof of Item $b$.
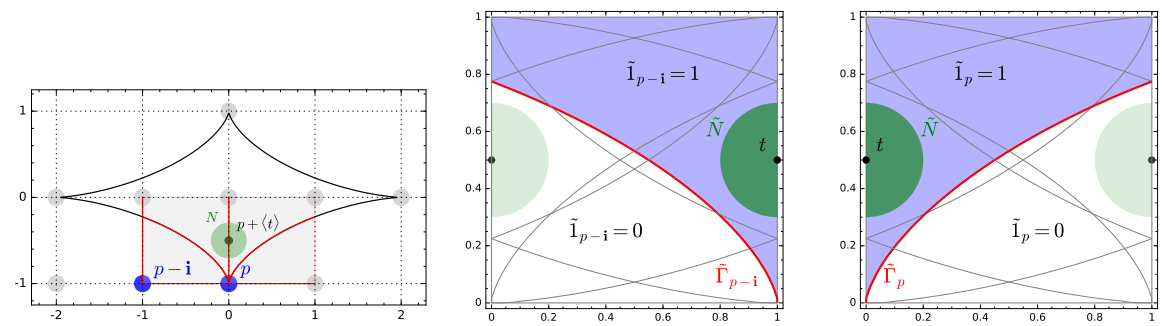

Figure 8: A point on the torus prime meridian link two successive indicator functions (Prop. 3.2-c).

In the next section, we use the previous results to propose two algorithms for the generation of the digitization classes. 


\section{Algorithms}

\subsection{Pointwise determination of the dual}

Starting from a set $S$ and a point $\mathbf{t} \in \mathbb{T}$, it is obviously possible to translate the set $S$ of $\mathbf{t}$ and to compute the corresponding Gauss digitization with one of the existing algorithms. The following algorithms show that it is possible to find any digitization by overlapping the grid squares $p \oplus[0,1)^{2}, p \in \mathbb{Z}^{2}$, containing the plot of the boundary of $S$ provided each square is labeled by its reference point $p$.

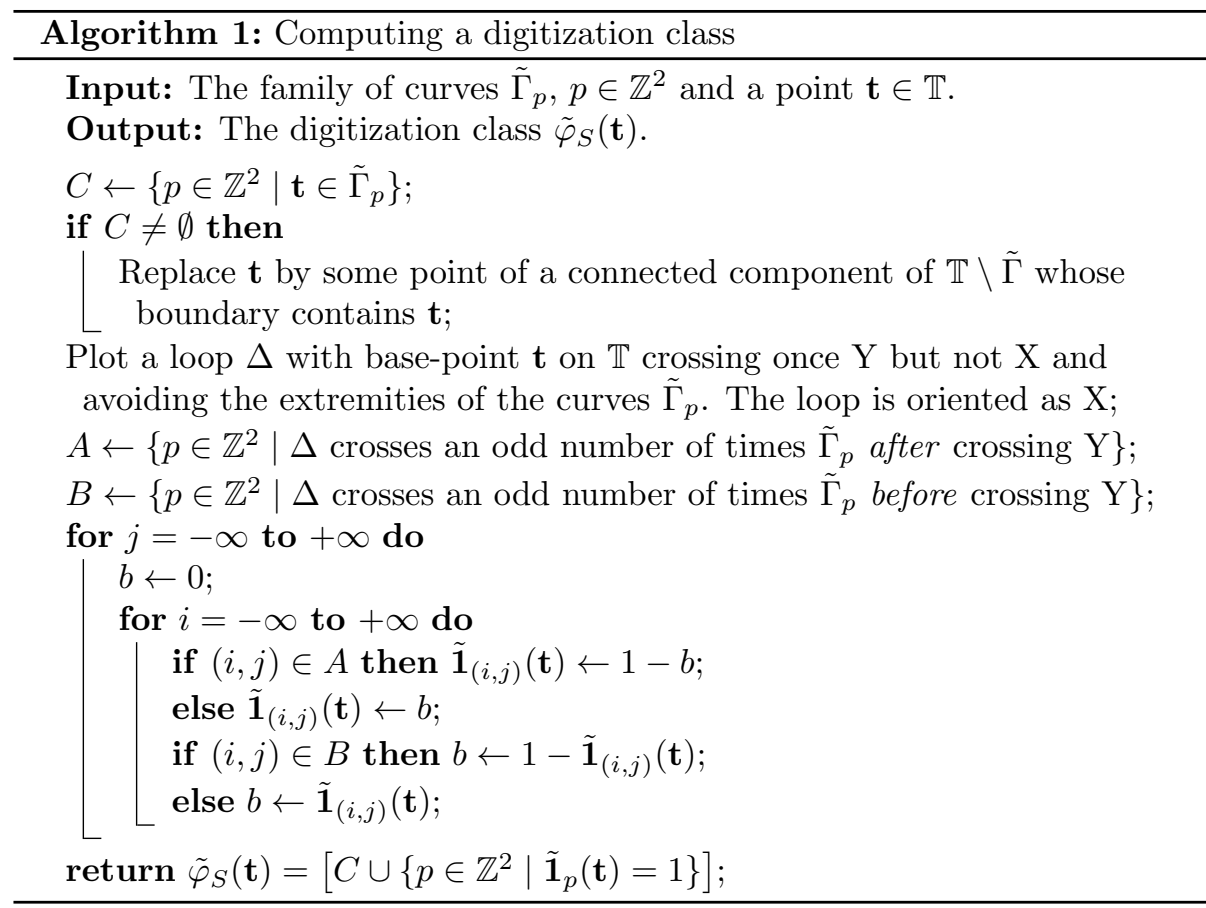

Proposition 4.1. Let $\tilde{\Gamma}$ be a contractile loop over the torus $\mathbb{T}$ and $S$ be a compact of $\mathbb{R}^{2}$ whose boundary projects on $\mathbb{T}$ as $\tilde{\Gamma}$. Then, for any translation $t$ over $\mathbb{R}^{2}$, Algorithm 1 provides the digitization of $t(S)$, up to an integer translation.

Proof. Recall that, for any $\mathbf{t} \in \mathbb{T}, \tilde{\varphi}_{S}(\mathbf{t})$ is a subset of $\mathbb{Z}^{2}$, that is a function from $\mathbb{Z}^{2}$ to $\{0,1\}$ whose value in $p \in \mathbb{Z}^{2}$ is $\tilde{\mathbf{1}}_{p}(\mathbf{t})$. Let $\mathbf{t}_{0}$ be the intersection point of $\Delta$ with the prime meridian. Let $j \in \mathbb{Z}(j$ is the common ordinate of the considered integer points). The proof is made by induction on the abscissa $i$ of the current integer point, $p=(i, j)$. We set $q=(i-1, j): q$ is the previous point in the induction. From Lemma $3.2-\mathrm{b}$, the value of $\tilde{\mathbf{1}}_{p}$ in $\mathbf{t}_{0}$ is equal to the value of $\tilde{\mathbf{1}}_{q}$ close to $\mathbf{t}_{0}$ and to the left of $\mathrm{Y}$. We note this value of $\tilde{\mathbf{1}}_{q}$ by $\tilde{\mathbf{1}}_{q}\left(t_{0}^{-}\right)$.

To initialize the induction, we observe that when $i$ is small enough, we clearly have $\tilde{\mathbf{1}}_{q}\left(\mathbf{t}_{0}^{-}\right)=b=0$. As induction hypothesis, we now assume that for some $i$, 
$b=\tilde{\mathbf{1}}_{q}\left(t_{0}^{-}\right)$. If $p \in A$, from Lemma 3.2-a we derive that $\tilde{\mathbf{1}}_{p}(t)=1-b$. Otherwise, $\tilde{\mathbf{1}}_{p}(t)=b$. This is the value of $\tilde{\mathbf{1}}_{p}(t)$ calculated by the algorithm. Alike, if $p \in B$, Lemma 3.2-b implies $\tilde{\mathbf{1}}_{p}\left(t_{0}^{-}\right)=1-\tilde{\mathbf{1}}_{p}(t)$. Otherwise, $\tilde{\mathbf{1}}_{p}\left(t_{0}^{-}\right)=\tilde{\mathbf{1}}_{p}(t)$. Then, in any case, the next value of $b$ computed by the algorithm is equal to $\tilde{\mathbf{1}}_{p}\left(t_{0}^{-}\right)$which achieves the induction.

In this algorithm, the value of $b$ propagates until a point in $A \cup B$ is encountered and this value codes for the membership of the points to the set. Then, when a point in $A \cup B$ is reached, depending whether the point is in $A \backslash B, B \backslash A$ or $A \cap B$, the value of $b$ is changed and the membership is revised. Figure 9 and Tab. 1 exemplifies Algorithm 1 on the set instance $S_{\text {astro }}$. 


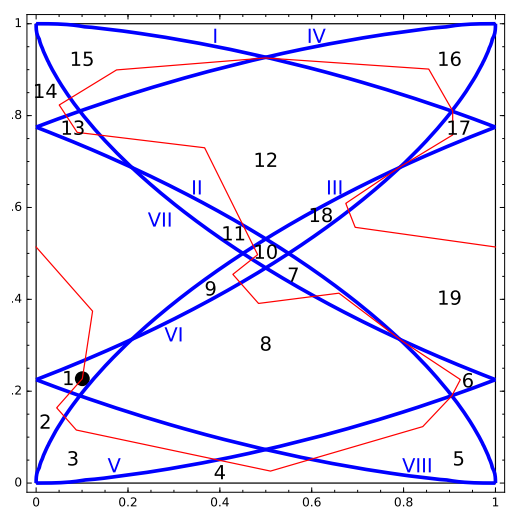

(a)

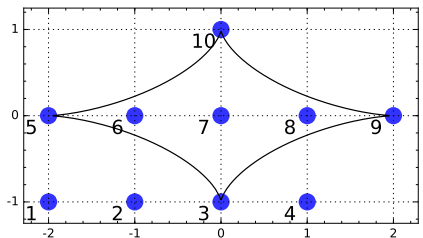

(b)

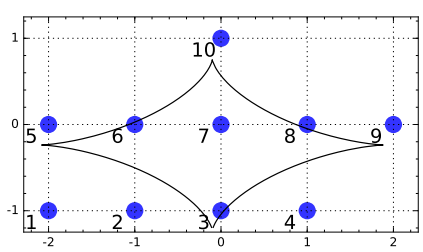

(c)

Figure 9: (Color online) (a) A loop (in red) whose base-point is the black point in the region 1 , runs through the connected components of $\mathbb{T} \backslash \tilde{\Gamma}$. The black Arabic numbers on the figure label these components according to the traveling order. The blue roman numbers label the arcs $\tilde{\Gamma}_{p}$ according to the numbering of the grid boundary points in (b). The ordered list of the curves $\tilde{\Gamma}_{p}$ crossed by the red loop before, resp. after, crossing the prime meridian is $[8,3,5,8,[2,5],[2,7], 7,6,7,3,2,7,4,7,[1,4],[1,6],[3,6], 6]$, resp. [6]. Then, in Algorithm $1, A=\{6\}$ and $B=\{2,3,7\}$. Table 1 is the trace of the execution of Algorithm 1. (c) The grid has been translated with the vector associated to the base point. The digitization now contains three points, numbered 3, 6, 7 . This is consistent with the output of Algorithm 1 shown in Table 1.

\begin{tabular}{cccccccc}
$j$ & $i$ & label & $A$ & $B$ & $b_{\text {in }}$ & $\tilde{\mathbf{1}}_{(i, j)}$ & $b_{\text {out }}$ \\
\hline-1 & -2 & 1 & & & 0 & 0 & 0 \\
& -1 & 2 & & $\mathrm{x}$ & 0 & 0 & 1 \\
& 0 & 3 & & $\mathrm{x}$ & 1 & 1 & 0 \\
& 1 & 4 & & & 0 & 0 & 0 \\
0 & -2 & 5 & & & 0 & 0 & 0 \\
& -1 & 6 & $\mathrm{x}$ & & 0 & 1 & 1 \\
& 0 & 7 & & $\mathrm{x}$ & 1 & 1 & 0 \\
& 1 & 8 & & & 0 & 0 & 0 \\
& 2 & 9 & & & 0 & 0 & 0 \\
1 & 0 & 10 & & & 0 & 0 & 0
\end{tabular}

Table 1: Values of the variables used in Algorithm 1 during the iteration process (if $\left.(i, j) \notin \mathcal{B}, b=\tilde{\mathbf{1}}_{(i, j)}=0\right)$. 


\subsection{Global determination of the dual}

Thanks to Lemma 3.2, we easily derive from Algorithm 1 a propagation algorithm that provides any digitization encountered when performing the torus loop.

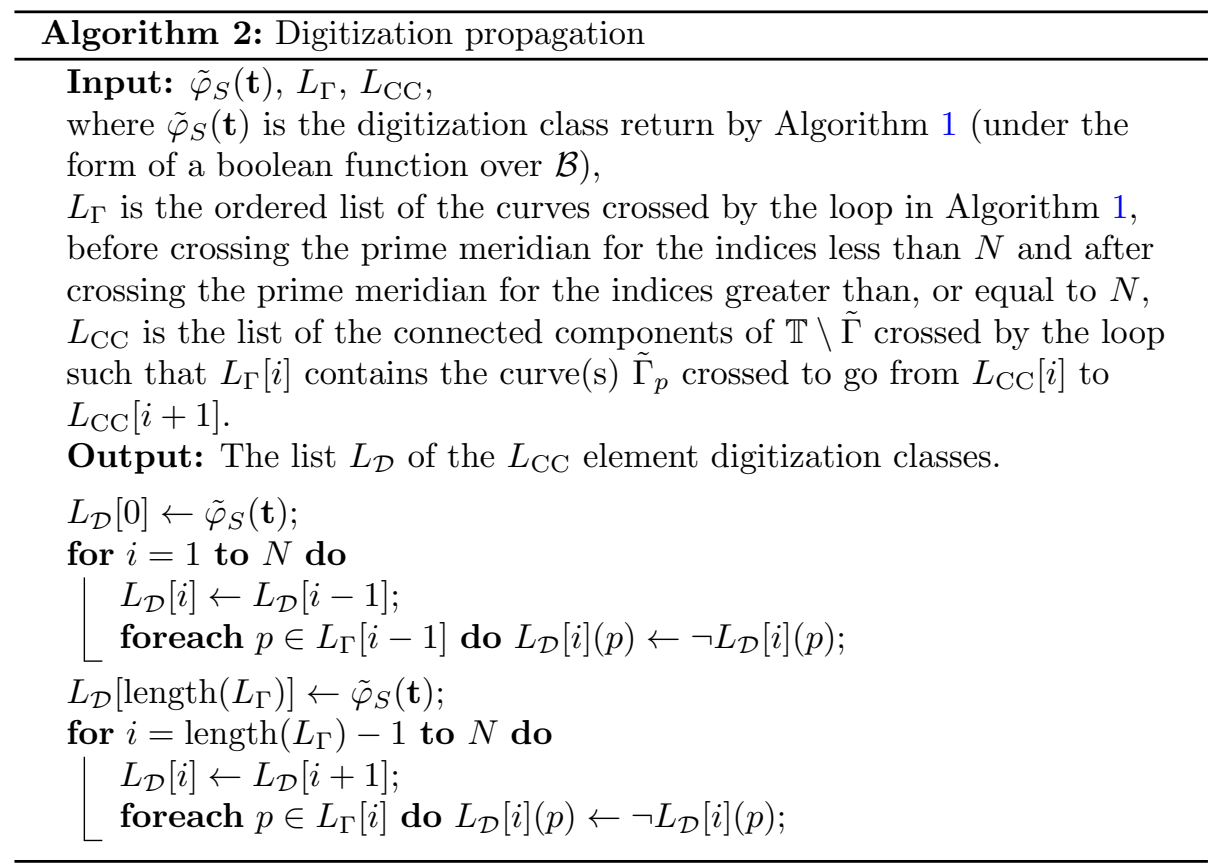

Table 2 shows the execution of Algorithm 2 on $S_{\text {astro }}$. One of the interest of Algorithm 2 is that there is no need to perform new scannings of the set $S$ once the first digitization have been computed. Only the points whose value changes from the current digitization to the next one are modified. 


\begin{tabular}{c|cc|cccccccccc} 
& \multicolumn{10}{|c|}{} & \multicolumn{10}{|c}{ grid boundary } \\
$i$ & $L_{\mathrm{CC}}[i]$ & $L_{\Gamma}[i-1]$ & 1 & 2 & 3 & 4 & 5 & 6 & 7 & 8 & 9 & 10 \\
\hline 0 & 1 & & 0 & 0 & 1 & 0 & 0 & 1 & 1 & 0 & 0 & 0 \\
1 & 2 & 8 & 0 & 0 & 1 & 0 & 0 & 1 & 1 & 1 & 0 & 0 \\
2 & 3 & 3 & 0 & 0 & 0 & 0 & 0 & 1 & 1 & 1 & 0 & 0 \\
3 & 4 & 5 & 0 & 0 & 0 & 0 & 1 & 1 & 1 & 1 & 0 & 0 \\
4 & 5 & 8 & 0 & 0 & 0 & 0 & 1 & 1 & 1 & 0 & 0 & 0 \\
5 & 6 & 2,5 & 0 & 1 & 0 & 0 & 0 & 1 & 1 & 0 & 0 & 0 \\
6 & 7 & 2,7 & 0 & 0 & 0 & 0 & 0 & 1 & 0 & 0 & 0 & 0 \\
7 & 8 & 7 & 0 & 0 & 0 & 0 & 0 & 1 & 1 & 0 & 0 & 0 \\
8 & 9 & 6 & 0 & 0 & 0 & 0 & 0 & 0 & 1 & 0 & 0 & 0 \\
9 & 10 & 7 & 0 & 0 & 0 & 0 & 0 & 0 & 0 & 0 & 0 & 0 \\
10 & 11 & 3 & 0 & 0 & 1 & 0 & 0 & 0 & 0 & 0 & 0 & 0 \\
11 & 12 & 2 & 0 & 1 & 1 & 0 & 0 & 0 & 0 & 0 & 0 & 0 \\
12 & 13 & 7 & 0 & 1 & 1 & 0 & 0 & 0 & 1 & 0 & 0 & 0 \\
13 & 14 & 4 & 0 & 1 & 1 & 1 & 0 & 0 & 1 & 0 & 0 & 0 \\
14 & 15 & 7 & 0 & 1 & 1 & 1 & 0 & 0 & 0 & 0 & 0 & 0 \\
15 & 16 & 1,4 & 1 & 1 & 1 & 0 & 0 & 0 & 0 & 0 & 0 & 0 \\
16 & 17 & 1,6 & 0 & 1 & 1 & 0 & 0 & 1 & 0 & 0 & 0 & 0 \\
17 & 18 & 3,6 & 0 & 1 & 0 & 0 & 0 & 0 & 0 & 0 & 0 & 0 \\
18 & 19 & 6 & 0 & 1 & 0 & 0 & 0 & 1 & 0 & 0 & 0 & 0 \\
$i$ & $L_{\mathrm{CC}}[i]$ & $L_{\Gamma}[i]$ & & & & & & & & & & \\
\hline 19 & 1 & & 0 & 0 & 1 & 0 & 0 & 1 & 1 & 0 & 0 & 0 \\
18 & 19 & 6 & 0 & 0 & 1 & 0 & 0 & 0 & 1 & 0 & 0 & 0
\end{tabular}

Table 2: Execution of Algorithm 2 on the output of Algorithm 1: the digitizations associated to the 19 visible regions of the dual of the set $S_{\text {astro }}$ are computed by propagation. The numbers in Columns 2 (regions) and 3 (points in $\mathcal{B}$ ) refer to the labeling of regions and points in Figure 9. Observe that we obtain two distinct digitizations for the region $19\left(L_{\mathrm{CC}}[18]\right)$ but they are in the same class of digitizations composed by two points vertically aligned (see Figure 2). 


\section{Conclusion}

We present in this paper a theoretical study on the digitizations obtained from a planar set under translation. The dual linking a translation vector class to a digitization class is defined. The dual is a piecewise constant function and the link between the dual constant region boundaries and the set frontier is established. The properties of the boundaries allow us to give a first algorithm generating a digitization from the labeled rigid body border and a second one generating all the digitizations from the labeled border and an initial digitization.

Future works on the digitization dual include the implementation of the proposed algorithms and the study of the dual under the (non-trivial) action of the rotation group and its combinatorial properties.

newpage

\section{Appendix A. Example}

Three dual examples are given in Figure A.10 for a complex object.

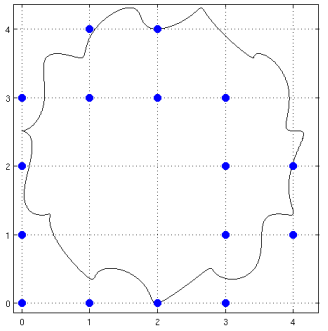

(a)

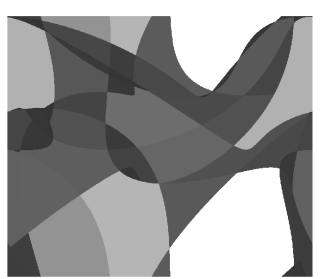

(d)

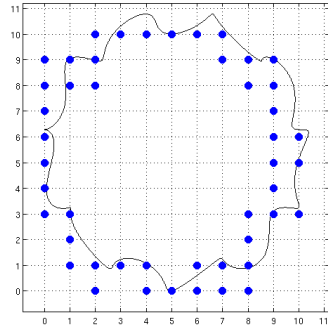

(b)

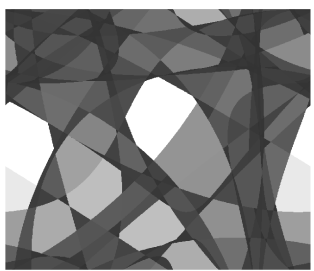

(e)

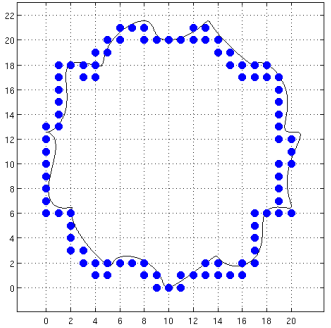

(c)

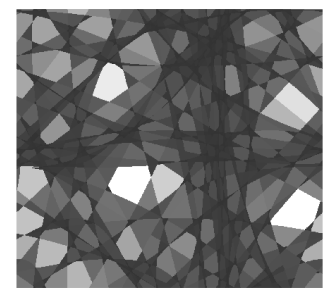

(f)

Figure A.10: Top: The frontier of a complex form digitized at 3 resolutions giving 3 grid boundaries with (a) 16, (b) 47 and (c) 93 points. Bottom: A representation of the corresponding piecewise constant dual functions where a each colored constant piece corresponds to a digitization. There are (d) 74, (e) 456 and (f) 1776 digitizations. The bigger the piece area, the whiter the gray shade. 


\section{References}

[BM16] Étienne Baudrier and Loïc Mazo. Curve digitization variability. In N. Normand, J. Guédon, and F. Autrusseau, editors, Proc 19th IAPR Int Conf on Discrete Geometry for Computer Imagery: DGCI 2016, volume 9647 of Lecture Notes in Computer Science, pages 59-70. Springer, 2016.

[DS84] Leo Dorst and Arnold W. M. Smeulders. Discrete Representation of Straight Lines. IEEE Transactions on Pattern Analysis and Machine Intelligence, PAMI-6(4):450-463, jul 1984.

[HŽ06] Martin N. Huxley and Jovisa Žunić. Different Digitisations of Displaced Discs. Foundations of Computational Mathematics, 6(2):255-268, apr 2006 .

[HŽ07] Martin N. Huxley and Jovisa Žunić. The number of N-point digital discs. IEEE Transactions on Pattern Analysis and Machine Intelligence, 29(1):159-161, 2007.

[HŽ16] Martin N. Huxley and Jovisa Žunić. The Number of Different Digital N-Discs. Journal of Mathematical Imaging and Vision, 56(3):403-408, 2016.

[Ken48] David G. Kendall. On the number of lattice points inside a random oval. The Quarterly Journal of Mathematics, 19:1-26, 1948.

[Nag05] Benedek Nagy. An algorithm to find the number of the digitizations of discs with a fixed radius. Electronic Notes in Discrete Mathematics, 20:607-622, 2005 .

[SK05] Peer Stelldinger and Ullrich Köthe. Towards a general sampling theory for shape preservation. Image Vision Comput., 23(2):237-248, 2005.

[TR02] Mohamed Tajine and Christian Ronse. Topological properties of Hausdorff discretization, and comparison to other discretization schemes. Theoretical Computer Science, 283(1):243-268, 2002. 